\title{
Throughput-Delay Trade-Off for Cognitive Radio Networks: A Convex Optimization Perspective
}

\author{
Hang Hu, Hang Zhang, and Hong Yu \\ College of Communications Engineering, PLA University of Science and Technology, Nanjing 210007, China \\ Correspondence should be addressed to Hang Zhang; hangzh_2002@163.com
}

Received 12 December 2013; Revised 15 February 2014; Accepted 12 March 2014; Published 7 April 2014

Academic Editor: Gerd Teschke

Copyright (C) 2014 Hang Hu et al. This is an open access article distributed under the Creative Commons Attribution License, which permits unrestricted use, distribution, and reproduction in any medium, provided the original work is properly cited.

\begin{abstract}
The throughput-delay trade-off problem for cooperative spectrum sensing (CSS) is investigated. It is proved that the maximum achievable throughput and the minimum transmission delay cannot be obtained simultaneously. An efficient algorithm is proposed to optimize the sensing bandwidth and the final decision threshold jointly such that the throughput is maximized while the delay is constrained. It is demonstrated that convex optimization plays an essential role in solving the problem in an efficient way. Simulation results show that the proposed optimal scheme can significantly improve the throughput of the secondary users (SUs) under the constraint that the delay Quality of Service (QoS) requirements of the SUs are satisfied.
\end{abstract}

\section{Introduction}

Cognitive radio $(\mathrm{CR})$ is a potential technology to improve the spectrum efficiency by allowing the secondary users to temporarily utilize the unused licensed spectrum of the primary users (PUs) [1,2]. Efficient and effective spectrum sensing is required to guarantee the QoS of both PU and SU [3-5]. To mitigate the impact of fading and shadowing, a variety of cooperative spectrum sensing methods (e.g., [610]) have been proposed. Authors in [11] consider CSS when two SUs collaborate via relaying scheme. In [12], the SU throughput of a sensing-based CR system with Markovian traffic is analyzed and optimized.

In the frame structure of CSS [13-15], the SU must cease transmission during the sensing slot. Thus, the transmission delay will be long, and the QoS will not be guaranteed for delay sensitive applications. To achieve continuous spectrum sensing, authors in [16-18] split the PU spectrum band into $K$ subbands and allocate one subband exclusively for detection purposes. In the new CSS frame structure shown in [19], the SUs conduct spectrum sensing and data transmission simultaneously over two different parts of the primary user spectrum band. In this way, the SUs do not need to cease transmission in the spectrum sensing stage, and the QoS can be guaranteed.

In this paper, we study the throughput-delay tradeoff problem for CSS with the new frame structure. It is proved that the maximum throughput and the minimum transmission delay cannot be obtained simultaneously. Our object is to design the sensing parameters to maximize the throughput of the SUs under the constraint that the delay QoS requirements of the SUs are satisfied.

We focus on optimizing the sensing bandwidth and the final decision threshold jointly such that the throughput is maximized while the delay is constrained. An efficient algorithm is proposed to obtain the optimal scheme. It is demonstrated that convex optimization plays an essential role in solving the associated design problems. Simulation results show that different delay QoS requirements require different values of sensing bandwidth. The throughput of the SUs can be greatly improved by using the proposed optimal scheme.

The rest of this paper is organized as follows. In Section 2, the mathematical formulation of the throughputdelay trade-off problem with new CSS frame structure is presented. Section 3 is devoted to the analysis of throughputdelay trade-off from a convex optimization perspective. Simulation results are provided in Section 4. Finally, in Section 5, we present the conclusion.

\section{Problem Formulation}

In CSS, consider the case that the SUs know the PU transmission bandwidth $W$. It was shown that authors in [19] proposed 


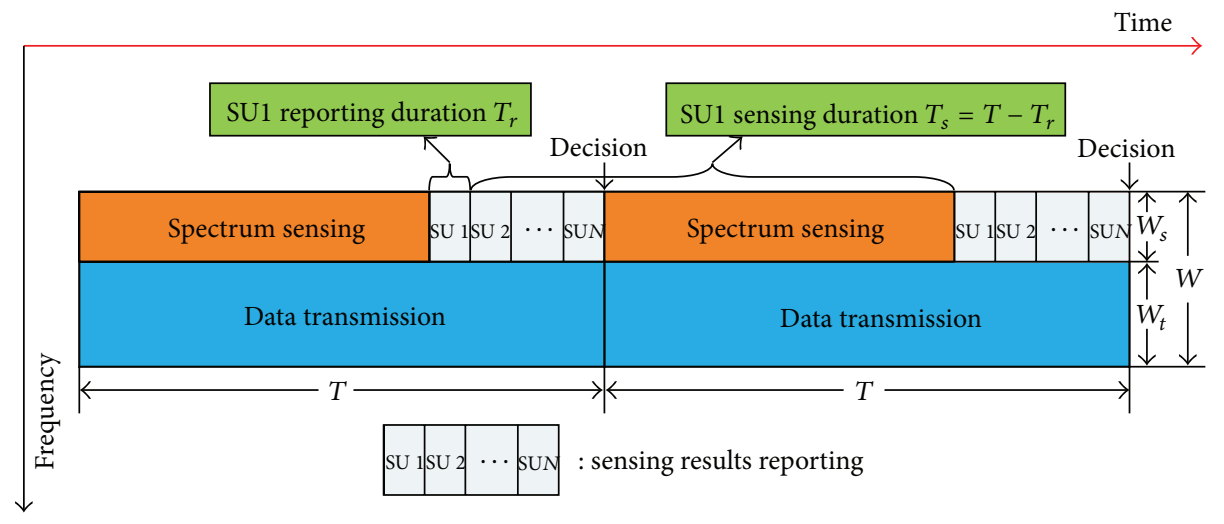

Figure 1: New frame structure for CSS.

a new CSS frame structure in which $W$ is divided into two parts. As depicted in Figure 1, $W_{s}$ is assigned exclusively for spectrum sensing and sensing results reporting, $W_{t}$ is assigned exclusively for potential SU data transmission, and $W_{s}+W_{t}=W$. To implement the new CSS frame structure, the SU transmitter is equipped with two radios, one for spectrum sensing and the other for data transmission. When the sensing result in the previous frame indicated that the PU was absent, the SU is allowed to transmit data. Otherwise, the SU keeps silent to protect the PU. Suppose that the frame duration is $T$ and the individual reporting duration is $T_{r}$. Since each SU continues spectrum sensing after sending its sensing result to the fusion center (FC), the sensing duration for each $\mathrm{SU}$ is $T_{s}=T-T_{r}$.

Considering that energy detection is employed to detect the status of PU in the local sensing step, the false alarm and detection probabilities at each SU are [18]

$$
\begin{gathered}
\mathscr{P}_{\mathrm{fa}}=Q\left(\frac{\epsilon}{\sqrt{2 T_{s} W_{s}}}-\sqrt{2 T_{s} W_{s}}\right), \\
\mathscr{P}_{\mathrm{de}}=Q\left(\frac{\epsilon}{(1+\gamma) \sqrt{2 T_{s} W_{s}}}-\sqrt{2 T_{s} W_{s}}\right),
\end{gathered}
$$

where $\epsilon$ is the energy detection threshold and $\gamma$ is the SNR of PU's signal at each SU. The false alarm probability is related to the detection probability as follows:

$$
\mathscr{P}_{\mathrm{fa}}=\mathbb{Q}\left((1+\gamma) \mathscr{Q}^{-1}\left(\mathscr{P}_{\mathrm{de}}\right)+\gamma \sqrt{2 T_{s} W_{s}}\right) .
$$

Suppose that the counting rule is employed in FC; then, the final false alarm and detection probabilities are [20]

$$
\begin{gathered}
Q_{\mathrm{fa}}=\sum_{i=k}^{N}\left(\begin{array}{c}
N \\
i
\end{array}\right)\left(\mathscr{P}_{\mathrm{fa}}\right)^{i}\left(1-\mathscr{P}_{\mathrm{fa}}\right)^{N-i}, \\
\mathrm{Q}_{\mathrm{de}}=\sum_{i=k}^{N}\left(\begin{array}{c}
N \\
i
\end{array}\right)\left(\mathscr{P}_{\mathrm{de}}\right)^{i}\left(1-\mathscr{P}_{\mathrm{de}}\right)^{N-i},
\end{gathered}
$$

where $N$ is the number of SUs and $k$ is the final decision threshold at FC.
There are two possible scenarios for which the SU can transmit its data. Scenario 1: the PU is correctly detected to be absent. The probability of scenario 1 happening is (1 $\left.Q_{\mathrm{fa}}\right) \mathscr{P}\left(H_{0}\right)$, where $\mathscr{P}\left(H_{0}\right)$ is the probability of absence of PU. In this scenario, the normalized achievable throughput of the SUs will be $\left(\left(W-W_{s}\right) / W\right) C$, where $C=\log _{2}\left(1+\gamma_{s}\right)$ and $\gamma_{s}$ is the SNR for the secondary link [21]. Scenario 2: the $\mathrm{PU}$ is falsely detected to be absent; that is, missed detection occurs, and the probability of this scenario happening is ( 1 $\left.Q_{\mathrm{de}}\right) \mathscr{P}\left(H_{1}\right)$, where $\mathscr{P}\left(H_{1}\right)$ is the probability of presence of PU. In this scenario, the channel is occupied simultaneously by the PU and the SU to transmit data, and the normalized achievable throughput of the SUs will be $\left(\left(W-W_{s}\right) / W\right) C_{1}$, where $C_{1}=\log _{2}\left(1+\left(\gamma_{s} /\left(1+\gamma_{p}\right)\right)\right)\left(\gamma_{p}=P_{p} / N_{0}\right.$, where $P_{p}$ is the interference power of the $\mathrm{PU}$ at the secondary receiver and $N_{0}$ is the noise power) [21]. The normalized achievable throughput of the SUs can be computed by

$$
\widehat{\mathscr{R}}=\mathscr{R}+\mathscr{R}_{1} \text {, }
$$

where

$$
\begin{gathered}
\mathscr{R}=\frac{W-W_{s}}{W} C\left(1-Q_{\mathrm{fa}}\right) \mathscr{P}\left(H_{0}\right), \\
\mathscr{R}_{1}=\frac{W-W_{s}}{W} C_{1}\left(1-Q_{\mathrm{de}}\right) \mathscr{P}\left(H_{1}\right) .
\end{gathered}
$$

In the new CSS frame structure, the SU transmission delay occurs in two cases: false alarm and correct detection of PU [18]. When false alarm happens, the transmission delay is

$$
\mathscr{D}_{1}=\mathscr{P}\left(H_{0}\right) Q_{\mathrm{fa}} \cdot T \text {. }
$$

In the case of correct detection of PU, the delay is

$$
\mathscr{D}_{2}=\mathscr{P}\left(H_{1}\right) Q_{\mathrm{de}} \cdot T \text {. }
$$

Thus, the normalized SU transmission delay is

$$
\mathscr{D}=\frac{\mathscr{D}_{1}+\mathscr{D}_{2}}{T}=\mathscr{P}\left(H_{0}\right) \mathrm{Q}_{\mathrm{fa}}+\mathscr{P}\left(H_{1}\right) \mathrm{Q}_{\mathrm{de}}
$$

To guarantee the QoS of the SUs, maximizing the throughput of the SUs $\widehat{R}$ while minimizing the SU transmission delay $\mathscr{D}$ subject to $Q_{\mathrm{de}} \geq Q_{\mathrm{de}}^{\text {th }}, 0<W_{s}<W$ and 
$1 \leq k \leq N$, may be of practical interest. Mathematically, the problem is given as

$$
\begin{array}{ll}
\max & \widehat{\mathscr{R}} \\
\min & \mathscr{D} \\
\text { s.t. } & Q_{\mathrm{de}} \geq Q_{\mathrm{de}}^{\text {th }} \\
& 0<W_{s}<W, \\
& 1 \leq k \leq N .
\end{array}
$$

For a given $W_{s}$ and $k$, we take the first partial derivative of $\widehat{\mathscr{R}}$ with respect to $Q_{\mathrm{de}}$ and obtain

$$
\begin{aligned}
& \frac{\partial \widehat{\mathscr{R}}}{\partial Q_{\mathrm{de}}}=-C \mathscr{P}\left(H_{0}\right) \frac{W-W_{s}}{W} \frac{d Q_{\mathrm{fa}}}{d Q_{\mathrm{de}}}-C_{1} \mathscr{P}\left(H_{1}\right) \frac{W-W_{s}}{W} \\
& =-C \mathscr{P}\left(H_{0}\right) \frac{W-W_{s}}{W} \frac{d Q_{\mathrm{fa}} / d \mathscr{P}_{\mathrm{de}}}{d Q_{\mathrm{de}} / d \mathscr{P}_{\mathrm{de}}} \\
& -C_{1} \mathscr{P}\left(H_{1}\right) \frac{W-W_{s}}{W} \\
& =-C \mathscr{P}\left(H_{0}\right) \frac{W-W_{s}}{W} \\
& \times \frac{N\left(\begin{array}{c}
N-1 \\
k-1
\end{array}\right) \mathscr{P}_{\mathrm{fa}}^{k-1}\left(1-\mathscr{P}_{\mathrm{fa}}\right)^{N-k}}{N\left(\begin{array}{c}
N-1 \\
k-1
\end{array}\right) \mathscr{P}_{\mathrm{de}}^{k-1}\left(1-\mathscr{P}_{\mathrm{de}}\right)^{N-k}} \frac{d \mathscr{P}_{\mathrm{fa}}}{d \mathscr{P}_{\mathrm{de}}} \\
& -C_{1} \mathscr{P}\left(H_{1}\right) \frac{W-W_{s}}{W} \\
& =-C \mathscr{P}\left(H_{0}\right) \frac{W-W_{s}}{W}\left(\frac{\mathscr{P}_{\mathrm{fa}}}{\mathscr{P}_{\mathrm{de}}}\right)^{k-1}\left(\frac{1-\mathscr{P}_{\mathrm{fa}}}{1-\mathscr{P}_{\mathrm{de}}}\right)^{N-k} \\
& \times \frac{d \mathscr{P}_{\mathrm{fa}} / d \epsilon}{d \mathscr{P}_{\mathrm{de}} / d \epsilon}-C_{1} \mathscr{P}\left(H_{1}\right) \frac{W-W_{s}}{W} \\
& =-C \mathscr{P}\left(H_{0}\right)(1+\gamma) \frac{W-W_{s}}{W}\left(\frac{\mathscr{P}_{\mathrm{fa}}}{\mathscr{P}_{\mathrm{de}}}\right)^{k-1} \\
& \times\left(\frac{1-\mathscr{P}_{\mathrm{fa}}}{1-\mathscr{P}_{\mathrm{de}}}\right)^{N-k} e^{(\epsilon \gamma /(1+\gamma))-\left(\epsilon^{2} \gamma(\gamma+2) / 4 T_{s} W_{s}(1+\gamma)^{2}\right)} \\
& -C_{1} \mathscr{P}\left(H_{1}\right) \frac{W-W_{s}}{W}<0 .
\end{aligned}
$$

Then, we take the first partial derivative of $\mathscr{D}$ with respect to $Q_{\mathrm{de}}$ and obtain

$$
\begin{aligned}
\frac{\partial \mathscr{D}}{\partial Q_{\mathrm{de}}} & =\mathscr{P}\left(H_{0}\right) \frac{d Q_{\mathrm{fa}}}{d Q_{\mathrm{de}}}+\mathscr{P}\left(H_{1}\right) \\
& =\mathscr{P}\left(H_{0}\right) \frac{d Q_{\mathrm{fa}} / d \mathscr{P}_{\mathrm{de}}}{d Q_{\mathrm{de}} / d \mathscr{P}_{\mathrm{de}}}+\mathscr{P}\left(H_{1}\right)
\end{aligned}
$$

$$
\begin{aligned}
= & \mathscr{P}\left(H_{0}\right) \frac{N\left(\begin{array}{c}
N-1 \\
k-1
\end{array}\right) \mathscr{P}_{\mathrm{fa}}^{k-1}\left(1-\mathscr{P}_{\mathrm{fa}}\right)^{N-k}}{N\left(\begin{array}{c}
N-1 \\
k-1
\end{array}\right) \mathscr{P}_{\mathrm{de}}^{k-1}\left(1-\mathscr{P}_{\mathrm{de}}\right)^{N-k}} \\
& \times \frac{d \mathscr{P}_{\mathrm{fa}}}{d \mathscr{P}_{\mathrm{de}}}+\mathscr{P}\left(H_{1}\right) \\
= & \mathscr{P}\left(H_{0}\right)\left(\frac{\mathscr{P}_{\mathrm{fa}}}{\mathscr{P}_{\mathrm{de}}}\right)^{k-1} \\
& \times\left(\frac{1-\mathscr{P}_{\mathrm{fa}}}{1-\mathscr{P}_{\mathrm{de}}}\right)^{N-k} \frac{d \mathscr{P}_{\mathrm{fa}} / d \epsilon}{d \mathscr{P}_{\mathrm{de}} / d \epsilon}+\mathscr{P}\left(H_{1}\right) \\
= & \mathscr{P}\left(H_{0}\right)(1+\gamma)\left(\frac{\mathscr{P}_{\mathrm{fa}}}{\mathscr{P}_{\mathrm{de}}}\right)^{k-1}\left(\frac{1-\mathscr{P}_{\mathrm{fa}}}{1-\mathscr{P}_{\mathrm{de}}}\right)^{N-k} \\
& \times e^{(\epsilon \gamma /(1+\gamma))-\left(\epsilon^{2} \gamma(\gamma+2) / 4 T_{s} W_{s}(1+\gamma)^{2}\right)}+\mathscr{P}\left(H_{1}\right) \\
> & 0 .
\end{aligned}
$$

Hence, $\widehat{\mathscr{R}}$ is a decreasing function of $Q_{\mathrm{de}}$, and $\mathscr{D}$ is an increasing function of $Q_{\mathrm{de}}$. This proves that the throughput of the SUs $\widehat{\mathscr{R}}$ is maximized and the SU transmission delay $\mathscr{D}$ is minimized only if the constraint (10) is at equality; that is, $Q_{\mathrm{de}}=Q_{\mathrm{de}}^{\text {th }}$. Therefore, the optimization problem can be reduced to

$$
\begin{array}{ll}
\max & \widehat{\mathscr{R}} \\
\min & \mathscr{D} \\
\text { s.t. } & Q_{\mathrm{de}}=Q_{\mathrm{de}}^{\text {th }}, \\
& 0<W_{s}<W, \\
& 1 \leq k \leq N .
\end{array}
$$

In scenario 2, the $\mathrm{CR}$ network experiences interference from the PU; obviously, $C>C_{1}$. Also, it is assumed that the probability of presence of PU is small, say less than 0.3 ; hence, it is economically advisable to explore the secondary usage for the PU frequency band. Also, to sufficiently protect the PU, the target detection probability $Q_{\mathrm{de}}^{\text {th }}$ is set not less than 0.9. Therefore, the optimization problem can be approximated by maximizing $\mathscr{R}$ while minimizing $\mathscr{D}$ subject to (16). Furthermore, in the next section, we will prove that the maximum throughput and the minimum delay cannot be obtained simultaneously. Therefore, the objective of throughput-delay trade-off is to design the sensing parameters such that the throughput $\mathscr{R}$ is maximized while the delay $\mathscr{D}$ is constrained. The problem formulation can be stated as follows:

$$
\begin{array}{ll}
\max & \mathscr{R} \\
\text { s.t. } & Q_{\mathrm{de}}=Q_{\mathrm{de}}^{\text {th }}, \\
& 0<W_{s}<W, \\
& 1 \leq k \leq N, \\
& \mathscr{D} \leq \mathscr{D}^{\text {th }},
\end{array}
$$

where $\mathscr{D}^{\text {th }}$ is the threshold of the delay constraint. 


\section{Throughput-Delay Trade-Off: A Convex Optimization Perspective}

In this section, we will investigate the throughput-delay trade-off problem from a convex optimization perspective. Let $\theta=(1+\gamma) \mathscr{Q}^{-1}\left(\mathscr{P}_{\mathrm{de}}\right)+\gamma \sqrt{2 T_{s} W_{s}}$, and since

$$
\frac{d \mathscr{P}_{\mathrm{fa}}}{d W_{s}}=-\frac{\gamma}{2} \sqrt{\frac{T_{s}}{\pi W_{s}}} e^{-\theta^{2} / 2}<0
$$

we have

$$
\begin{aligned}
\frac{\partial \mathscr{D}}{\partial W_{s}}= & \mathscr{P}\left(H_{0}\right) N\left(\begin{array}{c}
N-1 \\
k-1
\end{array}\right) \\
& \times\left(\mathscr{P}_{\mathrm{fa}}\right)^{k-1}\left(1-\mathscr{P}_{\mathrm{fa}}\right)^{N-k} \frac{d \mathscr{P}_{\mathrm{fa}}}{d W_{s}} \\
< & 0 .
\end{aligned}
$$

Thus, $\mathscr{D}$ is a decreasing function of $W_{s}$. Then,

$$
\frac{\partial \mathscr{R}}{\partial W_{s}}=-\frac{C}{W}\left(1-Q_{\mathrm{fa}}\right) \mathscr{P}\left(H_{0}\right)-\frac{W-W_{s}}{W} C \mathscr{P}\left(H_{0}\right) \frac{d Q_{\mathrm{fa}}}{d W_{s}},
$$

where

$$
\frac{d Q_{\mathrm{fa}}}{d W_{s}}=N\left(\begin{array}{c}
N-1 \\
k-1
\end{array}\right)\left(\mathscr{P}_{\mathrm{fa}}\right)^{k-1}\left(1-\mathscr{P}_{\mathrm{fa}}\right)^{N-k} \frac{d \mathscr{P}_{\mathrm{fa}}}{d W_{s}} .
$$

Obviously, $\lim _{W_{s} \rightarrow 0}\left(\partial \mathscr{R} / \partial W_{s}\right)=\infty, \lim _{W_{s} \rightarrow W}\left(\partial \mathscr{R} / \partial W_{s}\right)<$ 0 . Thus, there must exist an optimal value of $W_{s}$ that maximizes $\mathscr{R}$ and the root of $\partial \mathscr{R} / \partial W_{s}=0$ exists for $W_{s} \in$ $(0, W)$. Next, we will prove that the maximum point of $\mathscr{R}$ is unique; namely, the root of $\partial \mathscr{R} / \partial W_{s}=0$ is unique.

Substituting (18) and (21) into (20), we have

$$
\begin{aligned}
& \frac{\partial \mathscr{R}}{\partial W_{s}}=-\frac{C}{W}\left(1-Q_{\mathrm{fa}}\right) \mathscr{P}\left(H_{0}\right)+C \mathscr{P}\left(H_{0}\right) N\left(\begin{array}{c}
N-1 \\
k-1
\end{array}\right) \\
& \times\left(\mathscr{P}_{\mathrm{fa}}\right)^{k-1}\left(1-\mathscr{P}_{\mathrm{fa}}\right)^{N-k} \frac{\gamma}{2} \frac{W-W_{s}}{W} \sqrt{\frac{T_{s}}{\pi W_{s}}} e^{-\theta^{2} / 2} \\
& =-\frac{C}{W} \mathscr{P}\left(H_{0}\right)\left[\sum_{i=0}^{k-1}\left(\begin{array}{c}
N \\
i
\end{array}\right)\left(\mathscr{P}_{\mathrm{fa}}\right)^{i}\left(1-\mathscr{P}_{\mathrm{fa}}\right)^{N-i}\right. \\
& -N\left(\begin{array}{c}
N-1 \\
k-1
\end{array}\right)\left(\mathscr{P}_{\mathrm{fa}}\right)^{k-1}\left(1-\mathscr{P}_{\mathrm{fa}}\right)^{N-k} \\
& \left.\times\left(W-W_{s}\right) \frac{\gamma}{2} \sqrt{\frac{T_{s}}{\pi W_{s}}} e^{-\theta^{2} / 2}\right] .
\end{aligned}
$$

Setting $\partial \mathscr{R} / \partial W_{s}=0$, we have

$$
\begin{aligned}
\sum_{i=0}^{k-1}\left(\begin{array}{c}
N \\
i
\end{array}\right)\left(\mathscr{P}_{\mathrm{fa}}\right)^{i}\left(1-\mathscr{P}_{\mathrm{fa}}\right)^{N-i} \\
=N\left(\begin{array}{c}
N-1 \\
k-1
\end{array}\right)\left(\mathscr{P}_{\mathrm{fa}}\right)^{k-1}\left(1-\mathscr{P}_{\mathrm{fa}}\right)^{N-k} \\
\quad \times\left(W-W_{s}\right) \frac{\gamma}{2} \sqrt{\frac{T_{s}}{\pi W_{s}}} e^{-\theta^{2} / 2} .
\end{aligned}
$$

Then,

$$
\begin{aligned}
& \frac{\sum_{i=0}^{k-1}\left(\begin{array}{c}
N \\
i
\end{array}\right)\left(\mathscr{P}_{\mathrm{fa}}\right)^{i}\left(1-\mathscr{P}_{\mathrm{fa}}\right)^{N-i}}{N\left(\begin{array}{c}
N-1 \\
k-1
\end{array}\right)\left(\mathscr{P}_{\mathrm{fa}}\right)^{k-1}\left(1-\mathscr{P}_{\mathrm{fa}}\right)^{N-k}\left(W-W_{s}\right)(\gamma / 2) \sqrt{T_{s} / \pi W_{s}}} \\
& \quad=e^{-\theta^{2} / 2},
\end{aligned}
$$

$$
\ln \left[\frac{\sum_{i=0}^{k-1}\left(\begin{array}{c}
N \\
i
\end{array}\right)\left(\mathscr{P}_{\mathrm{fa}}\right)^{i-k+1}\left(1-\mathscr{P}_{\mathrm{fa}}\right)^{k-i}}{N\left(\begin{array}{c}
N-1 \\
k-1
\end{array}\right)\left(W-W_{s}\right)(\gamma / 2) \sqrt{T_{s} / \pi W_{s}}}\right]=-\frac{1}{2} \theta^{2} .
$$

According to (25), it can be derived that $u=v$, where

$$
\begin{gathered}
u=\ln \left[\frac{2(k-1) !(N-k) !}{\gamma\left(W-W_{s}\right) N !} \sqrt{\frac{\pi W_{s}}{T_{s}}}\right. \\
\left.\times \sum_{i=0}^{k-1}\left(\begin{array}{c}
N \\
i
\end{array}\right)\left(\mathscr{P}_{\mathrm{fa}}\right)^{i-k+1}\left(1-\mathscr{P}_{\mathrm{fa}}\right)^{k-i}\right], \\
v=-\frac{1}{2} \theta^{2} .
\end{gathered}
$$

Taking the first derivative of $u$ with respect to $W_{s}$, it is derived that

$$
\frac{d u}{d W_{s}}=\phi_{1}+\phi_{2}+\phi_{3}
$$

where

$$
\begin{gathered}
\phi_{1}=\frac{W+W_{s}}{2 W_{s}\left(W-W_{s}\right)}, \\
\phi_{2}=-\frac{d \mathscr{P}_{\mathrm{fa}}}{d W_{s}} \frac{\sum_{i=0}^{k-1}\left(\begin{array}{c}
N \\
i
\end{array}\right)(k-1-i)\left(\mathscr{P}_{\mathrm{fa}}\right)^{i-k}\left(1-\mathscr{P}_{\mathrm{fa}}\right)^{k-i}}{\sum_{i=0}^{k-1}\left(\begin{array}{c}
N \\
i
\end{array}\right)\left(\mathscr{P}_{\mathrm{fa}}\right)^{i-k+1}\left(1-\mathscr{P}_{\mathrm{fa}}\right)^{k-i}}, \\
\phi_{3}=-\frac{d \mathscr{P}_{\mathrm{fa}}}{d W_{s}} \frac{\sum_{i=0}^{k-1}\left(\begin{array}{c}
N \\
i
\end{array}\right)(k-i)\left(\mathscr{P}_{\mathrm{fa}}\right)^{i-k+1}\left(1-\mathscr{P}_{\mathrm{fa}}\right)^{k-i-1}}{\sum_{i=0}^{k-1}\left(\begin{array}{c}
N \\
i
\end{array}\right)\left(\mathscr{P}_{\mathrm{fa}}\right)^{i-k+1}\left(1-\mathscr{P}_{\mathrm{fa}}\right)^{k-i}} .
\end{gathered}
$$

Since $\phi_{1}>0, \phi_{2}>0$, and $\phi_{3}>0$, we have $d u / d W_{s}>0$, and $u$ is an increasing function of $W_{s}$. 


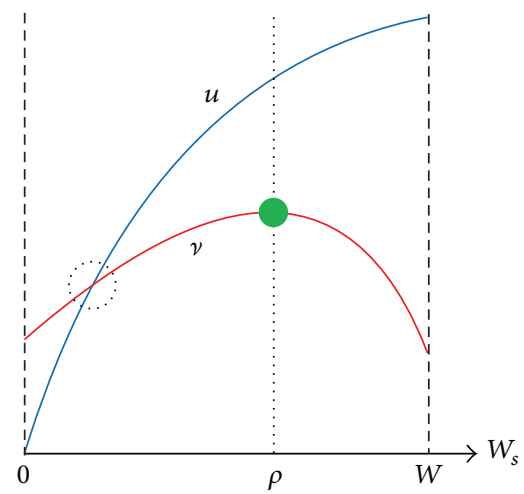

(a)

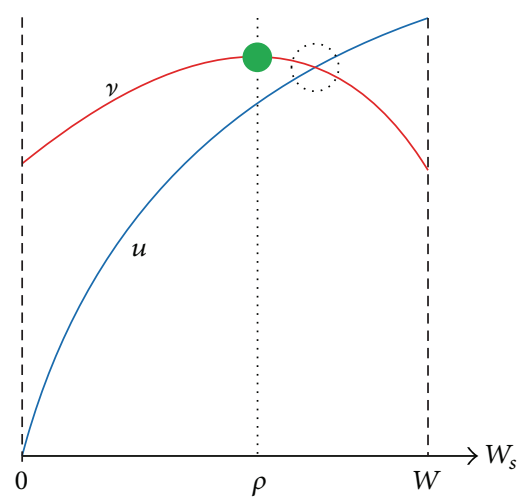

(b)

FIGURE 2: Illustration of the curves: $u$ and $v$.

Taking the first derivative of $v$ with respect to $W_{s}$, it is derived that

$$
\begin{aligned}
\frac{d v}{d W_{s}} & =-\theta \frac{d \theta}{d W_{s}} \\
& =-\left((1+\gamma) Q^{-1}\left(\mathscr{P}_{\mathrm{de}}\right)+\gamma \sqrt{2 T_{s} W_{s}}\right) \gamma \sqrt{\frac{T_{s}}{2 W_{s}}} .
\end{aligned}
$$

Let $\rho=\left((1+\gamma) \mathscr{Q}^{-1}\left(\mathscr{P}_{\mathrm{de}}\right)\right)^{2} / 2 T_{s} \gamma^{2}$, for $W_{s} \in(0, \rho], d v / d W_{s} \geq$ 0 ; for $W_{s} \in(\rho, W), d v / d W_{s}<0$. Thus, $v$ is an increasing function of $W_{s}$ for $W_{s} \in(0, \rho]$ and a decreasing function of $W_{s}$ for $W_{s} \in(\rho, W)$. We have proved that $d u / d W_{s}>d v / d W_{s}$ for $W_{s} \in(0, \rho]$ in the Appendix.

We know that $u$ and $v$ must intersect each other since the root of $\partial \mathscr{R} / \partial W_{s}=0$ exists for $W_{s} \in(0, W)$. If $u$ and $v$ have an intersection for $W_{s} \in(0, \rho]$, since $u$ increases faster than $v$ for $W_{s} \in(0, \rho]$ and $u>v$ for $W_{s} \in(\rho, W)$, there is only one intersection between $u$ and $v$, which is illustrated in Figure 2(a). If $u$ and $v$ do not have an intersection for $W_{s} \in(0, \rho]$, they must have an intersection for $W_{s} \in(\rho, W)$. Since $u$ is an increasing function of $W_{s}$ and $v$ is a decreasing function of $W_{s}$ for $W_{s} \in(\rho, W)$, there is only one intersection between $u$ and $v$, which is illustrated in Figure 2(b). Thus, the root of $\partial \mathscr{R} / \partial W_{s}=0$ is unique.

From the above analysis, we can conclude that $\mathscr{R}$ is a unimodal function for $W_{s} \in(0, W)$. We define $W_{s}^{*}$ as the root of $\partial \mathscr{R} / \partial W_{s}=0$. Since $\mathscr{D}$ is a decreasing function of $W_{s}$, there

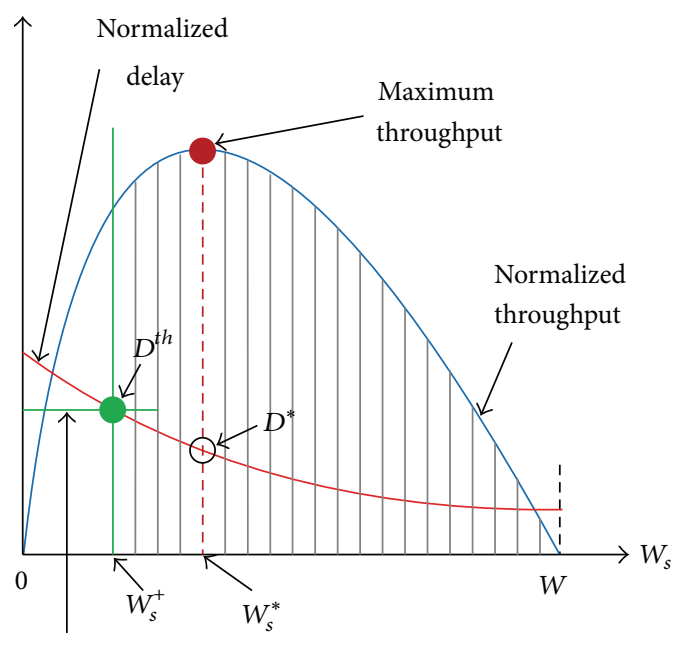

Delay constraint

(a)

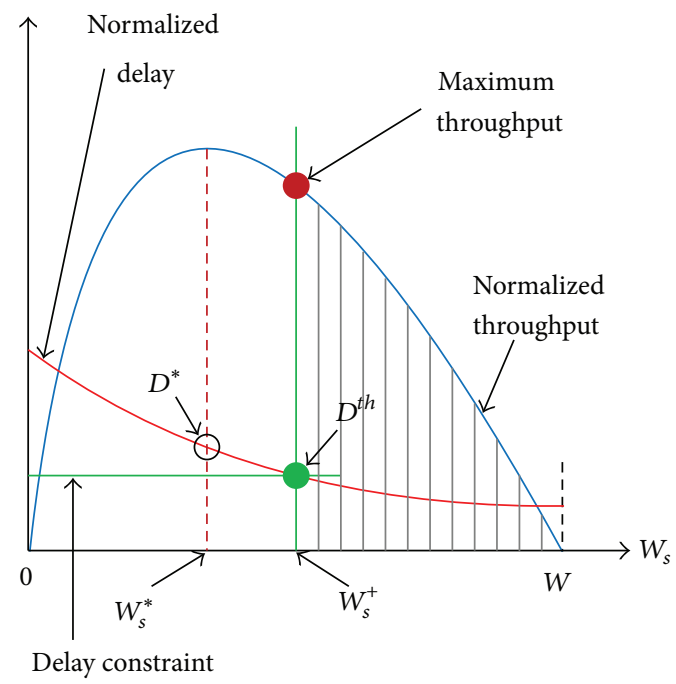

(b)

FIgURE 3: Illustration of the curves: normalized throughput $\mathscr{R}$ and normalized delay $\mathscr{D}$.

is no value of $W_{s}$ that can maximize the throughput $\mathscr{R}$ and minimize the delay $\mathscr{D}$ simultaneously. We will optimize $W_{s}$ and $k$ jointly such that the throughput is maximized while the delay is constrained. To satisfy the delay QoS requirement, we set $\mathscr{D} \leq \mathscr{D}^{\text {th }}$, where $\mathscr{D}^{\text {th }}$ is the threshold of the delay constraint. Since $\mathscr{D}$ is a decreasing function of $W_{s}$, we should choose $W_{s} \geq W_{s}^{+}$, where $W_{s}^{+}$is the minimum sensing bandwidth that can satisfy the delay QoS requirement and is determined by $\mathscr{D}^{\text {th }}$.

Different SUs have different QoS requirements. Let $\mathscr{D}^{*}$ denote the transmission delay which is corresponding to $W_{s}^{*}$. For the SUs with relaxed delay QoS requirements, $\mathscr{D}^{\text {th }}$ may be larger than $\mathscr{D}^{*}$ and $W_{s}^{+}<W_{s}^{*}$. In this case, we should choose $W_{s}^{*}$ to maximize the throughput $\mathscr{R}$, which is illustrated in Figure 3(a). For the SUs with stringent delay QoS requirements, $\mathscr{D}^{\text {th }}$ may be smaller than or equal to 


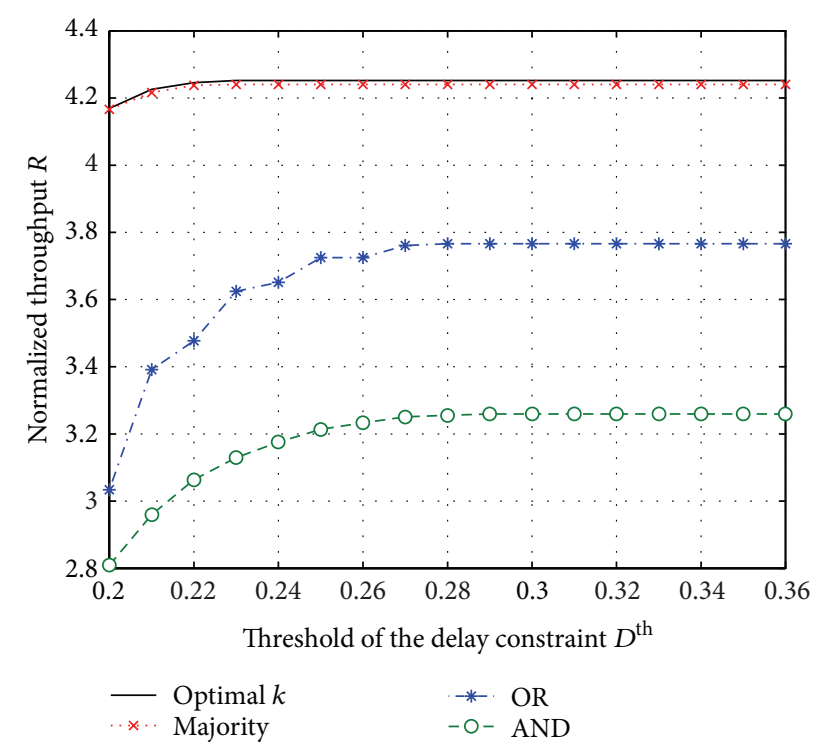

FIGURE 4: Normalized throughput $\mathscr{R}$ for various counting rules.

$\mathscr{D}^{*}$ and $W_{s}^{+} \geq W_{s}^{*}$. In this case, we should choose $W_{s}^{+}$ to maximize the throughput $\mathscr{R}$, which is illustrated in Figure $3(\mathrm{~b})$. For the optimal final decision threshold $k_{\text {opt }}$, no closed-form solution can be obtained. Hence, we will search through $k$ from 1 to $N$ to obtain $k_{\text {opt }}$. The optimal scheme that maximizes the throughput $\mathscr{R}$ can be divided into 3 steps as follows.

Step 1. For each $k(k=1,2, \ldots, N)$, calculate the root $\mathscr{P}_{\mathrm{de}, k}$ of $\sum_{i=k}^{N}\left(\begin{array}{c}N \\ i\end{array}\right)\left(\mathscr{P}_{d e}\right)^{i}\left(1-\mathscr{P}_{\mathrm{de}}\right)^{N-i}=Q_{\mathrm{de}}^{\text {th }}$, the root $W_{s, k}^{*}$ of $\partial \mathscr{R} / \partial W_{s}=0$, and the root $\mathscr{P}_{\mathrm{fa}, k}$ of $Q_{\mathrm{fa}}=\left(\mathscr{D}^{\text {th }}-\right.$ $\left.\mathscr{P}\left(H_{1}\right) Q_{\mathrm{de}}^{\text {th }}\right) / \mathscr{P}\left(H_{0}\right)$ by using the Bisection method [22].

Step 2. According to $\mathscr{P}_{\mathrm{de}, k}$ and $\mathscr{P}_{\mathrm{fa}, k}$, calculate $W_{s, k}^{+}$. If $W_{s, k}^{*}>$ $W_{s, k}^{+}$, choose optimal sensing bandwidth $W_{s, k}^{\mathrm{opt}}=W_{s, k}^{*}$; otherwise, choose $W_{s, k}^{\mathrm{opt}}=W_{s, k}^{+}$.

Step 3. Calculate and compare $\mathscr{R}_{k}$, and choose the maximum one.

\section{Simulation Results}

To evaluate the throughput-delay trade-off for various sensing schemes, simulation results have been conducted in this section. The frame duration is $T=20 \mathrm{~ms}$; the individual reporting duration is $T_{r}=1 \mathrm{~ms}$; the $\mathrm{PU}$ transmission bandwidth is $W=2.5 \times 10^{4} \mathrm{~Hz}$; the number of SUs is $N=9$; the SNR of the PU's signal at the receiver of SU is $\gamma=-10 \mathrm{~dB}$ unless otherwise stated; the SNR for the secondary link is $\gamma_{s}=20 \mathrm{~dB} ; \mathscr{P}\left(H_{0}\right)=0.8$ unless otherwise stated.

Figure 4 illustrates the normalized throughput $\mathscr{R}$ versus the threshold of the delay constraint for various counting rules. Optimal $W_{s}$ is employed and $Q_{\mathrm{de}}^{\text {th }}=0.9$. When $\mathscr{D}^{\text {th }}$ initially increases, the throughput increases. This means that relaxing the delay constraint will result in a higher

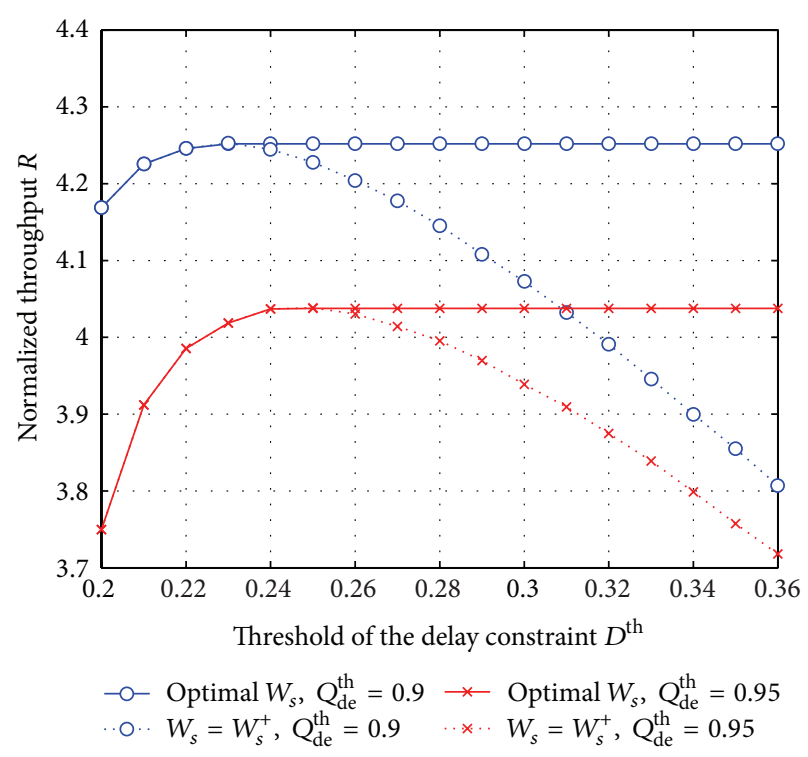

FIGURE 5: Normalized throughput $\mathscr{R}$ for various sensing schemes.

throughput. However, when $\mathscr{D}^{\text {th }}$ is increased further, the throughput no longer increases and remains unchanged since the optimal sensing bandwidth is $W_{s}^{*}$ in this case, and the throughput reaches the peak value. It is also seen that the optimal scheme can achieve a higher throughput than that using fixed thresholds. Majority rule is suboptimal and AND rule performs the worst. Thus, to enhance the throughput of the SUs, the final decision threshold $k$ needs to be optimized.

In Figure 5, the optimal $k$ values are used for each sensing scheme. For the SUs with stringent delay QoS requirements, $\mathscr{D}^{\text {th }} \leq \mathscr{D}^{*}$, it can be observed that the throughput with optimal $W_{s}$ is the same as that using $W_{s}^{+}$as the sensing bandwidth. This is because the optimal $W_{s}$ is equal to $W_{s}^{+}$ in this case, which has been discussed in Section 3. For the SUs with relaxed delay QoS requirements, $\mathscr{D}^{\text {th }}>\mathscr{D}^{*}$, the throughput with optimal $W_{s}$ reaches the peak value and remains unchanged. However, the throughput with $W_{s}=W_{s}^{+}$ decreases as the threshold of the delay constraint increases. This is because the optimal $W_{s}$ is equal to $W_{s}^{*}$ and $W_{s}^{+}<W_{s}^{*}$ in this case, which has been discussed in Section 3. Larger $Q_{\mathrm{de}}^{\text {th }}$ means better protection to PU. It is seen that relaxing the constraint on the protection of PU will result in a higher throughput.

In Figures 6 and 7, the sensing bandwidth $W_{s}$ and the final decision threshold $k$ are jointly optimized, $Q_{\mathrm{de}}^{\mathrm{th}}=0.95$. Figure 6 is simulated to show the normalized throughput $\mathscr{R}$ versus the threshold of the delay constraint with different values of $\mathscr{P}\left(H_{0}\right)$. It is also observed that when $\mathscr{D}^{\text {th }}$ initially increases, the throughput $\mathscr{R}$ increases. However, when $\mathscr{D}^{\text {th }}$ is increased further $\left(\mathscr{D}^{\text {th }}>\mathscr{D}^{*}\right)$, the throughput $\mathscr{R}$ reaches the peak value and remains unchanged. The larger the value of $\mathscr{P}\left(H_{0}\right)$, the higher the normalized throughput $\mathscr{R}$; this is because more spectrum opportunities can be reused by the SUs. In addition, $\mathscr{D}^{*}$ decreases as $\mathscr{P}\left(H_{0}\right)$ becomes larger. In Figure 7, it is shown that the larger the value of $\gamma$, the higher 


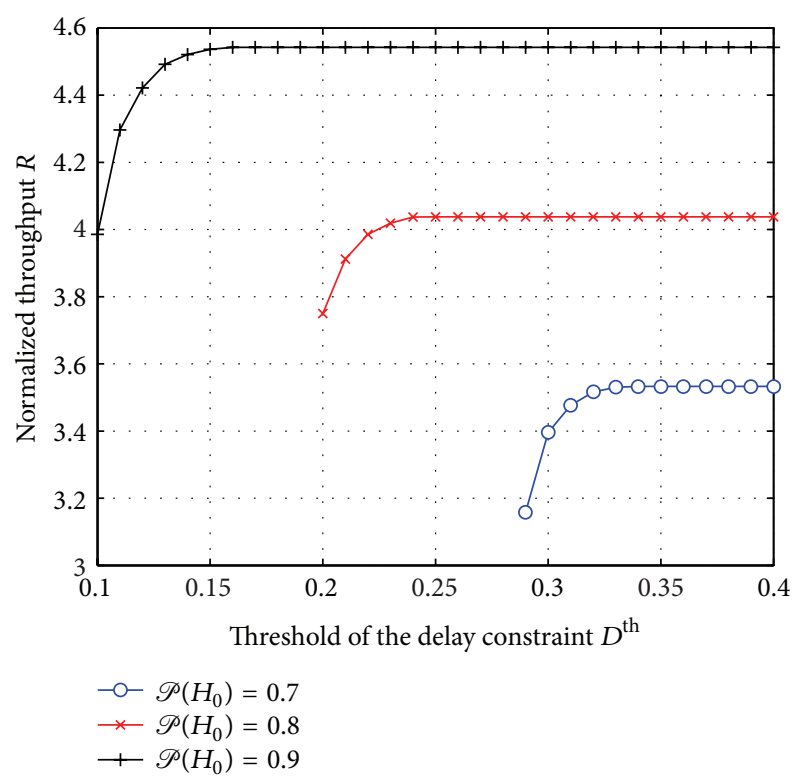

FIGURE 6: Normalized throughput $\mathscr{R}$ versus threshold of the delay constraint with different values of $\mathscr{P}\left(H_{0}\right)$.

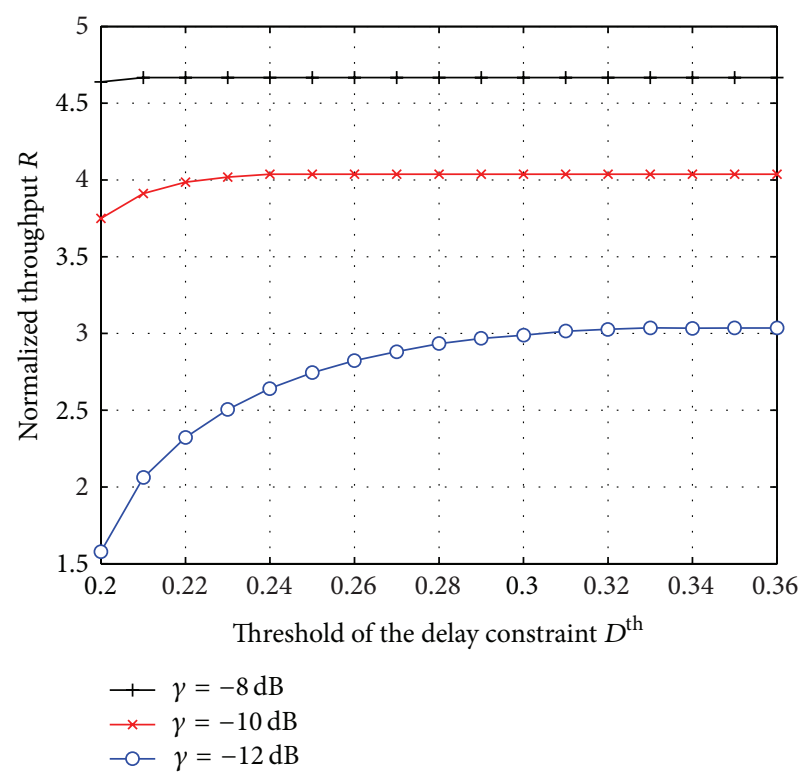

FIGURE 7: Normalized throughput $\mathscr{R}$ versus threshold of the delay constraint with different values of $\gamma$.

the normalized throughput $\mathscr{R}$. In addition, $\mathscr{D}^{*}$ decreases as the SNR of the PU's signal at the receiver of SU increases.

\section{Conclusion}

This paper studies the new CSS frame structure and formulates the throughput-delay trade-off problem. Particularly, we optimize the sensing bandwidth and the final decision threshold jointly to maximize the throughput of the SUs under the constraint that the delay QoS requirements of the SUs are satisfied. And we have demonstrated the key role of convex optimization in solving the associated design problems. Simulation results have shown that different delay QoS requirements require different optimal $W_{s}$ values, and the optimal scheme can effectively improve the throughput of the SUs.

\section{Appendix}

\section{Proof of $d u / d W_{s}>d v / d W_{s}$ for $W_{s} \in(0, \rho]$}

According to (27) and (29), we have $d u / d W_{s}=\phi_{1}+\phi_{2}+\phi_{3}$, $d v / d W_{s}=-\gamma \sqrt{T_{s} / 2 W_{s}} \cdot \theta$, where $\phi_{1}, \phi_{2}, \phi_{3}$, and $\theta$ are defined in Section 3. Obviously,

$$
\frac{d u}{d W_{s}}>\phi_{3}>-\frac{d \mathscr{P}_{\mathrm{fa}}}{d W_{s}} \frac{1}{1-\mathscr{P}_{f a}}=\frac{\gamma}{2} \sqrt{\frac{T_{s}}{\pi W_{s}}} \frac{e^{-\theta^{2} / 2}}{\mathscr{Q}(-\theta)} .
$$

According to [23], $e^{-x^{2} / 2} / \mathbb{Q}(x)>\sqrt{2 \pi} x$ for $x \geq 0$. For $W_{s} \in(0, \rho], \theta \leq 0$; then, $-\theta \geq 0$ and $e^{-\theta^{2} / 2} / \mathbb{Q}(-\theta)>\sqrt{2 \pi}(-\theta)$. Thus,

$$
\frac{\gamma}{2} \sqrt{\frac{T_{s}}{\pi W_{s}}} \frac{e^{-\theta^{2} / 2}}{Q(-\theta)}>\gamma \sqrt{\frac{T_{s}}{2 W_{s}}}(-\theta)=\frac{d v}{d W_{s}} .
$$

Therefore, $d u / d W_{s}>d v / d W_{s}$ for $W_{s} \in(0, \rho]$.

\section{Conflict of Interests}

The authors declare that there is no conflict of interests regarding the publication of this paper.

\section{Acknowledgments}

This work is supported by the National Basic Research Program of China (973 no. 2009CB3020400) and the Jiangsu Province Natural Science Foundation under Grant BK2011002.

\section{References}

[1] J. Mitola III and G. Q. Maguire Jr., "Cognitive radio: making software radios more personal," IEEE Personal Communications, vol. 6, no. 4, pp. 13-18, 1999.

[2] S. Haykin, "Cognitive radio: brain-empowered wireless communications," IEEE Journal on Selected Areas in Communications, vol. 23, no. 2, pp. 201-220, 2005.

[3] W. Yin, P. Ren, and C. Zhang, "A joint sensing-time adaption and data transmission scheme in cognitive radio networks," in Proceedings of the 54th Annual IEEE Global Telecommunications Conference (GLOBECOM '11), pp. 1-5, Houston, Tex, USA, December 2011.

[4] S. Stotas and A. Nallanathan, "On the throughput and spectrum sensing enhancement of opportunistic spectrum access cognitive radio networks," IEEE Transactions on Wireless Communications, vol. 11, no. 1, pp. 97-107, 2012.

[5] E. C. Y. Peh, Y.-C. Liang, and Y. Zeng, "Sensing and power control in cognitive radio with location information," in Proceedings of the IEEE International Conference on Communication Systems (ICCS '12), pp. 255-259, Singapore, 2012. 
[6] T. Yücek and H. Arslan, "A survey of spectrum sensing algorithms for cognitive radio applications," IEEE Communications Surveys \& Tutorials, vol. 11, no. 1, pp. 116-130, 2009.

[7] D. Duan, L. Yang, and J. C. Principe, "Cooperative diversity of spectrum sensing for cognitive radio systems," IEEE Transactions on Signal Processing, vol. 58, no. 6, pp. 3218-3227, 2010.

[8] J. Shen, S. Liu, L. Zeng, G. Xie, J. Gao, and Y. Liu, "Optimisation of cooperative spectrum sensing in cognitive radio network," IET Communications, vol. 3, no. 7, pp. 1170-1178, 2009.

[9] W. Zhang, R. K. Mallik, and K. Ben Letaief, "Optimization of cooperative spectrum sensing with energy detection in cognitive radio networks," IEEE Transactions on Wireless Communications, vol. 8, no. 12, pp. 5761-5766, 2009.

[10] S. Atapattu, C. Tellambura, and H. Jiang, "Energy detection based cooperative spectrum sensing in cognitive radio networks," IEEE Transactions on Wireless Communications, vol. 10, no. 4, pp. 1232-1241, 2011.

[11] T. Cui, F. Gao, and A. Nallanathan, "Optimization of cooperative spectrum sensing in cognitive radio," IEEE Transactions on Vehicular Technology, vol. 60, no. 4, pp. 1578-1589, 2011.

[12] G. Noh, J. Lee, H. Wang, S. Kim, S. Choi, and D. Hong, "Throughput analysis and optimization of sensing-based cognitive radio systems with Markovian traffic," IEEE Transactions on Vehicular Technology, vol. 59, no. 8, pp. 4163-4169, 2010.

[13] E. C. Y. Peh, Y.-C. Liang, Y. L. Guan, and Y. Pei, "Energy-efficient cooperative spectrum sensing in cognitive radio networks," in Proceedings of the 54th Annual IEEE Global Telecommunications Conference (GLOBECOM '11), pp. 1-5, Houston, Tex, USA, December 2011.

[14] X. Feng, X. Gan, and X. Wang, "Energy-constrained cooperative spectrum sensing in cognitive radio networks," in Proceedings of the 54th Annual IEEE Global Telecommunications Conference (GLOBECOM '11), pp. 1-5, Houston, Tex, USA, December 2011.

[15] Y. Gao, W. Xu, K. Yang, K. Niu, and J. Lin, "Energy efficient transmission with cooperative spectrum sensing in cognitive radionetworks," in Proceedings of the IEEE Wireless Communications and Networking Conference (WCNC 13), pp. 7-12, Shanghai, China, 2013.

[16] G. Ganesan, Y. Li, B. Bing, and S. Li, "Spatiotemporal sensing in cognitive radio networks," IEEE Journal on Selected Areas in Communications, vol. 26, no. 1, pp. 5-12, 2008.

[17] H. Li, H. Dai, and C. Li, "Collaborative quickest spectrum sensing via random broadcast in cognitive radio systems," IEEE Transactions on Wireless Communications, vol. 9, no. 7, pp. 23382348, 2010.

[18] W. Yin, P. Ren, Q. Du, and Y. Wang, "Delay and throughput oriented continuous spectrum sensing schemes in cognitive radio networks," IEEE Transactions on Wireless Communications, vol. 11, no. 6, pp. 2148-2159, 2012.

[19] H. Hu, H. Zhang, and H. Yu, "Delay QoS guaranteed cooperativespectrum sensing in cognitive radio networks," International Journal of Electronics and Communications, vol. 67, no. 9, pp. 804-807, 2013.

[20] W. Han, J. Li, Z. Tian, and Y. Zhang, "Efficient cooperative spectrum sensing with minimum overhead in cognitive radio," IEEE Transactions on Wireless Communications, vol. 9, no. 10, pp. 3006-3011, 2010.

[21] Y.-C. Liang, Y. Zeng, E. C. Y. Peh, and A. T. Hoang, "Sensingthroughput tradeoff for cognitive radio networks," IEEE Transactions on Wireless Communications, vol. 7, no. 4, pp. 1326-1337, 2008.
[22] S. C. Chapra and R. P. Canale, Numerical Methods for Engineers, McGraw-Hill, New York, NY, USA, 6th edition, 2010.

[23] N. Kingsbury, "Approximation Formulae for the Gaussian Error Integral, Q(x)," Connexions, 2005, http://cnx.org/content/ m11067/2.4/. 


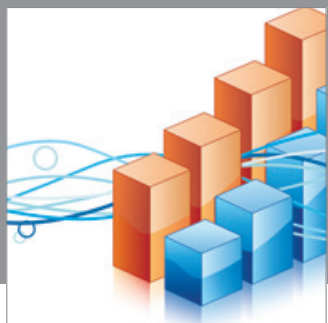

Advances in

Operations Research

mansans

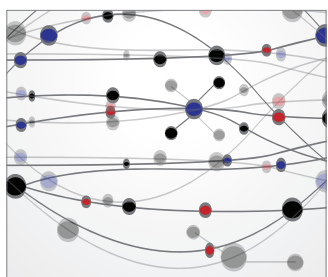

The Scientific World Journal
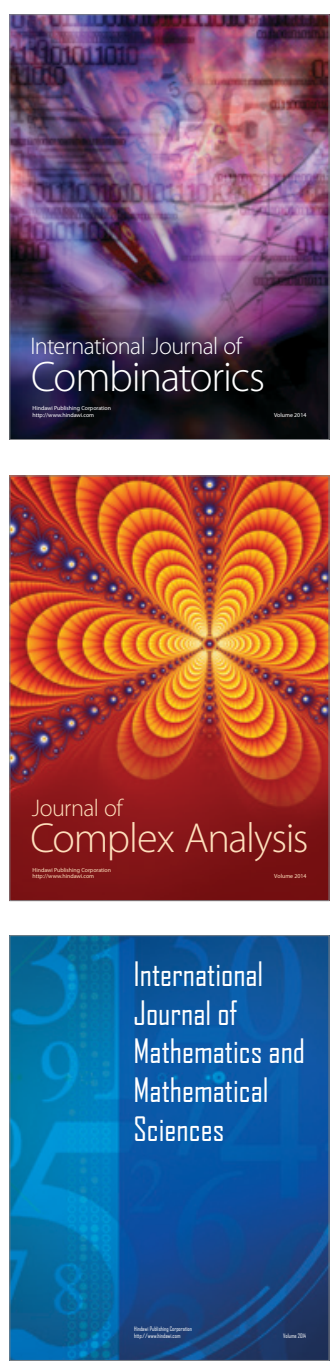
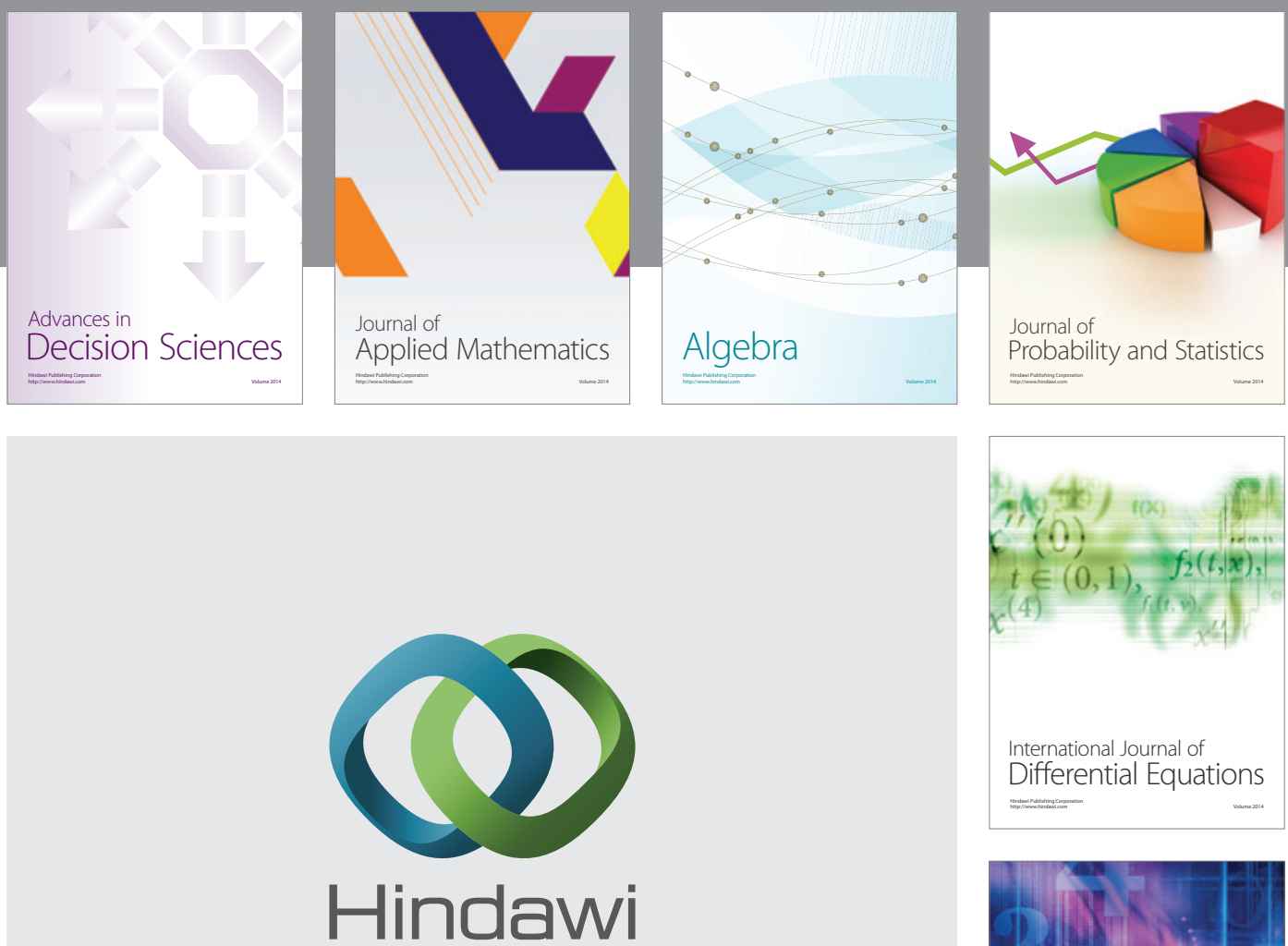

Submit your manuscripts at http://www.hindawi.com
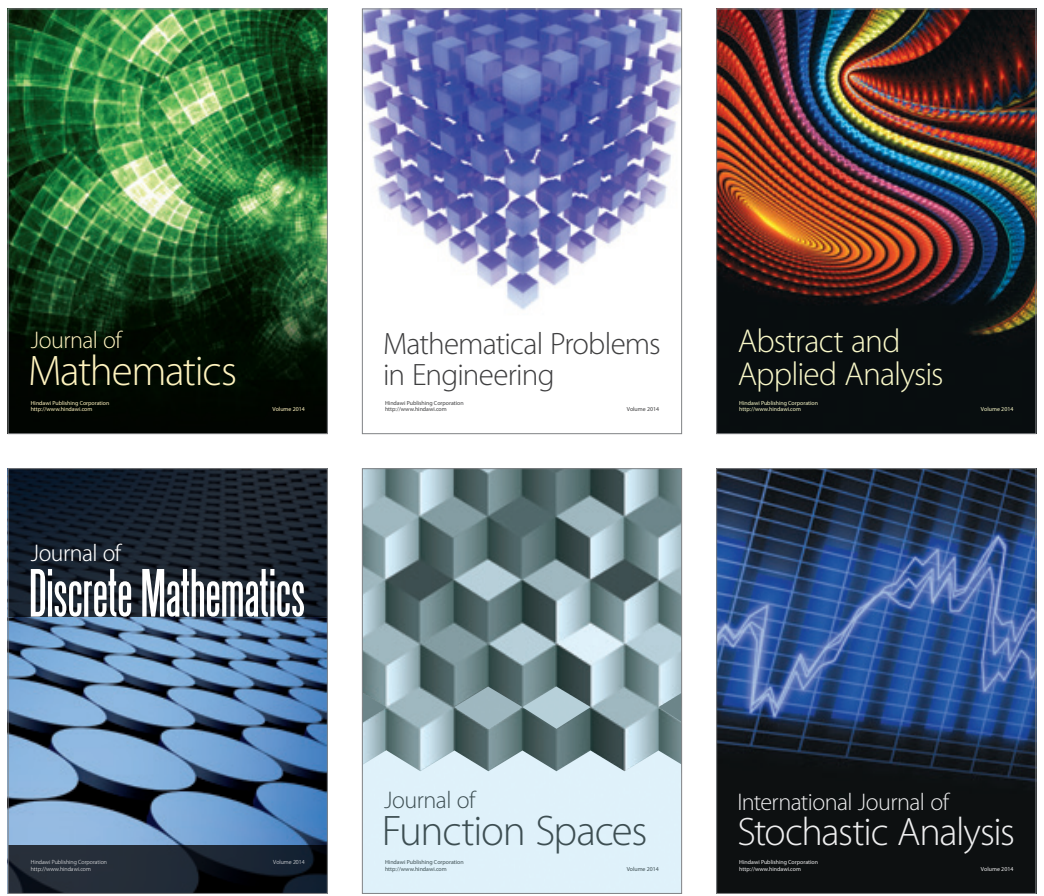

Journal of

Function Spaces

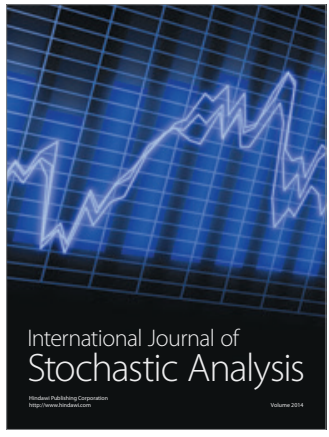

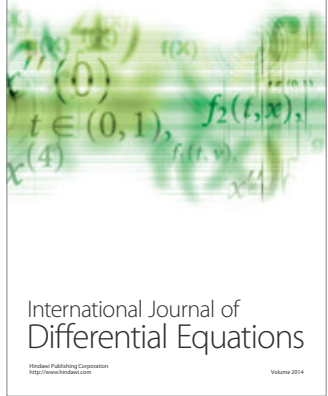
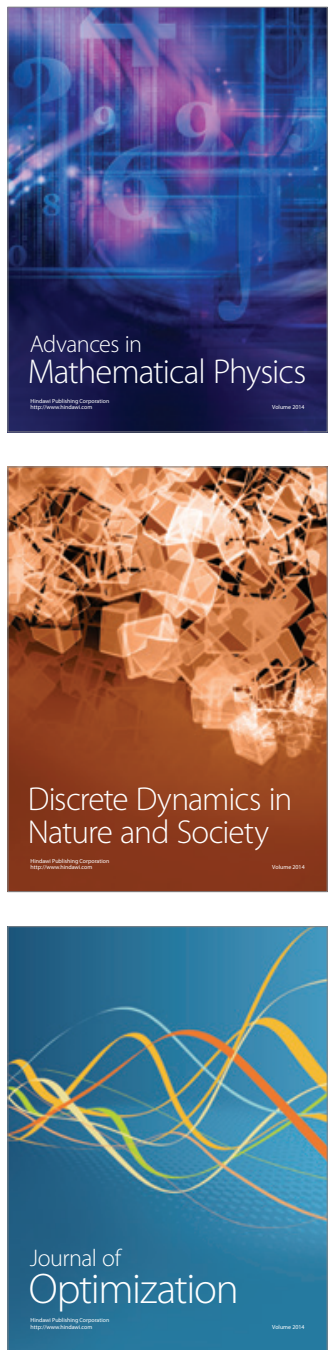